\title{
MOTION OF CHARGED PARTICLES IN TWO-STEP NILPOTENT LIE GROUPS
}

\author{
OSAMU IKAWA
}

Communicated by Charles-Michel Marle

Abstract. We formulate the equation of motion of a charged particle in a Riemannian manifold with a closed two form. Since a two-step nilpotent Lie group has natural left-invariant closed two forms, it is natural to consider the motion of a charged particle in a simply connected two-step nilpotent Lie groups with a left invariant metric. We study the behavior of the motion of a charged particle in the above spaces.

\section{Introduction}

Let $\Omega$ be a closed two-form on a connected Riemannian manifold $(M,\langle\rangle$,$) , where$ $\langle$,$\rangle is a Riemannian metric on M$. We denote by $\bigwedge^{m}(M)$ the space of $m$-forms on $M$. We denote by $\iota(X): \bigwedge^{m}(M) \rightarrow \bigwedge^{m-1}(M)$ the interior product operator induced from a vector field $X$ on $M$, and by $\mathcal{L}: T(M) \rightarrow T^{*}(M)$, the Legendre transformation from the tangent bundle $T(M)$ over $M$ onto the cotangent bundle $T^{*}(M)$ over $M$, which is defined by

$$
\mathcal{L}: T(M) \rightarrow T^{*}(M), \quad u \mapsto \mathcal{L}(u), \quad \mathcal{L}(u)(v)=\langle u, v\rangle, \quad u, v \in T(M) .
$$

A curve $x(t)$ in $M$ is referred as a motion of a charged particle under electromagnetic field $\Omega$, if it satisfies the following second order differential equation

$$
\nabla_{\dot{x}} \dot{x}=-\mathcal{L}^{-1}(\iota(\dot{x}) \Omega)
$$

where $\nabla$ is the Levi-Civita connection of $M$. Here $\nabla_{\dot{x}} \dot{x}$ means the acceleration of the charged particle. Since $-\mathcal{L}^{-1}(\iota(\dot{x}) \Omega)$ is perpendicular to the direction $\dot{x}$ of the movement, $-\mathcal{L}^{-1}(\iota(\dot{x}) \Omega)$ means the Lorentz force. The speed $|\dot{x}|$ is a conservative constant for a charged particle. When $\Omega=0$, then the motion of a charged particle is nothing but a geodesic. The equation (2) originated in the theory of relativity (see [2] for details).

In this paper, we deal with the motion of a charged particles in a simply connected two-step nilpotent Lie group $N$ with a left invariant Riemannian metric. 
Since a two-step nilpotent Lie group has a non-trivial center $Z$, we can construct a left-invariant closed two form $\Omega_{a_{0}}$ from an element $a_{0} \in Z$ specified below and consider the motion of a charged particle under the electromagnetic field $\Omega_{a_{0}}$. H. Naitoh and Y. Sakane proved that there are no closed geodesics in a simply connected nilpotent Lie group. In contrast with geodesics, there exist motions of charged particles which are periodic. Kaplan defined a $H$-type Lie group, which is a kind of two-step nilpotent Lie groups. We study the motion of a charged particle in a $H$-type Lie group more explicitly than in a general two-step nilpotent Lie group.

\section{Charged Particles in Two-step Nilpotent Lie Groups}

Let $N$ be a simply connected two-step nilpotent Lie group with a left-invariant Riemannian metric $\langle$,$\rangle . Denote by \mathfrak{n}$ the vector space consisting of all left-invariant vector fields on $N$. Since $\mathfrak{n}$ is two-step nilpotent, $\mathfrak{n}$ has a non-trivial center $\mathfrak{z}$. Let $\mathfrak{n}=\mathfrak{z} \oplus \mathfrak{z}^{\perp}$ be an orthogonal direct sum decomposition of $\mathfrak{n}$, then $\left[\mathfrak{z}^{\perp}, \mathfrak{z}^{\perp}\right] \subset \mathfrak{z}$. For $a_{0} \in \mathfrak{z}$, we define a linear transformation $\phi_{a_{0}}$ on $\mathfrak{z}^{\perp}$ by

$$
\left\langle\phi_{a_{0}}(X), Y\right\rangle=\left\langle a_{0},[X, Y]\right\rangle, \quad X, Y \in \mathfrak{z}^{\perp} .
$$

We extend $\phi_{a_{0}}$ to a linear transformation on $\mathfrak{n}$ by $\phi=0$ on $\mathfrak{z}$, which is also denoted by $\phi_{a_{0}}$. We can regard $\phi_{a_{0}}$ as a left-invariant $(1,1)$-tensor on $N$. Then $\phi_{a_{0}}$ is skew-symmetric with respect to the left-invariant Riemannian metric $\langle$,$\rangle since$

$$
\left\langle\phi_{a_{0}}(X), Y\right\rangle+\left\langle X, \phi_{a_{0}}(Y)\right\rangle=\left\langle a_{0},[X, Y]\right\rangle+\left\langle a_{0},[Y, X]\right\rangle=0
$$

for any left invariant vector fields $X, Y \in \mathfrak{n}$. Define a left-invariant two-form $\Omega_{a_{0}}$ on $N$ by

$$
\Omega_{a_{0}}(X, Y)=\left\langle X, \phi_{a_{0}}(Y)\right\rangle, \quad X, Y \in \mathfrak{n}
$$

then a simple calculation implies that $\Omega_{a_{0}}$ is closed. In fact, for any $X_{1}, X_{2}$ and $X_{3}$ in $\mathfrak{n}$ we have

$$
\begin{aligned}
3 !\left(\mathrm{d} \Omega_{a_{0}}\right)\left(X_{1}, X_{2}, X_{3}\right) & =-\mathfrak{S} \Omega_{a_{0}}\left(\left[X_{1}, X_{2}\right], X_{3}\right) \\
& =-\mathfrak{S}\left\langle\left[X_{1}, X_{2}\right], \phi_{a_{0}}\left(X_{3}\right)\right\rangle=0
\end{aligned}
$$

where we denote by $\mathfrak{S}$ the cyclic sum, and the last equality follows from the fact that $\left[X_{1}, X_{2}\right] \in \mathfrak{z}$ and $\phi\left(X_{3}\right) \in \mathfrak{z}^{\perp}$. The equation of motion of the charged particle under the electromagnetic field $\Omega_{a_{0}}$ is

$$
\nabla_{\dot{x}} \dot{x}=\phi_{a_{0}}(\dot{x}) .
$$


Here a curve in a manifold is simple if it is a simply closed periodic curve, or if it does not intersect itself. Since $N$ is simply connected, the one dimensional de-Rham cohomology group vanishes. Hence we get the following theorem using Theorem 9 in [2].

Theorem 1. The motion of a charged particle (3) in a simply connected two-step nilpotent Lie group is simple.

Now we will construct explicitly a simply connected two step nilpotent Lie group with a left-invariant Riemannian metric from an (abstract) two-step nilpotent Lie algebra $\mathfrak{n}$ with an inner product $\langle$,$\rangle . In order to do this, we recall a Hausdorff$ formula for a Lie group (see [1, p. 106]), which states that

$$
\exp X \exp Y=\exp \left(X+Y+\frac{1}{2}[X, Y]+\cdots\right) \text {. }
$$

If the Lie group is two-step nilpotent, then the higher terms $+\cdots$ on the right hand side vanish. Based on the Hausdorff formula, we define a Lie group structure on $\mathfrak{n}$ itself by

$$
X \cdot Y=X+Y+\frac{1}{2}[X, Y], \quad X, Y \in \mathfrak{n} .
$$

The identity element in this group is 0 , and the inverse element of $x \in \mathfrak{n}$ is equal to $-x$. We denote by $N=(\mathfrak{n}, \cdot)$ the so obtained Lie group. The center of $N$ coincides with $\mathfrak{z}$. Denote by $\operatorname{Lie}(N)$ the Lie algebra consisting of all left-invariant vector fields on $N$. Then $\operatorname{Lie}(N)$ is identified with $\mathfrak{n}$ as a Lie algebra as mentioned below. Since $N$ is a Euclidean space as a manifold, we can identify $T_{0}(N)$ with $\mathfrak{n}$ as vector spaces. The identification induces a Lie algebra structure on $T_{0}(N)$. For $X \in T_{0}(N)$, we denote by $\tilde{X} \in \operatorname{Lie}(N)$ the left-invariant vector field on $N$ such that $\tilde{X}_{0}=X$. The mapping defined by $\mathfrak{n}=T_{0}(N) \rightarrow \operatorname{Lie}(N), X \mapsto \tilde{X}$ gives an isomorphism as Lie algebras. Hence $N$ is a simply connected two-step nilpotent Lie group whose Lie algebra is $\mathfrak{n}$. The inner product $\langle$,$\rangle on \mathfrak{n}$ induces a left-invariant Riemannian metric $\langle$,$\rangle on N$. Using this notation, we have

$$
\Omega_{a_{0}}(\tilde{X}, \tilde{Y})=\langle\tilde{X}, \phi \tilde{Y}\rangle=\left\langle\tilde{a_{0}},[\tilde{Y}, \tilde{X}]\right\rangle=\left\langle a_{0},[Y, X]\right\rangle .
$$

The exponential mapping exp $: \mathfrak{n} \rightarrow N$ is equal to identity mapping. Hence for $X \in T_{0}(N)$, we have

$$
\tilde{X}_{x}=\frac{\mathrm{d}}{\mathrm{d} t}(x \cdot t X)_{\mid t=0}=\frac{\mathrm{d}}{\mathrm{d} t}\left(x+t X+\frac{t}{2}[x, X]\right)_{\mid t=0} \in T_{x}(N) .
$$

Since the Riemannian metric on $N$ is left-invariant, the left action of $N$ on $N$ itself is isometric. Hence $X \in T_{0}(N)$ induces a Killing vector field $X^{*}$ on $N$ by

$$
X_{x}^{*}=\frac{\mathrm{d}}{\mathrm{d} t}(\exp t X) x_{\mid t=0}=\frac{\mathrm{d}}{\mathrm{d} t}\left(t X+x+\frac{t}{2}[X, x]\right)_{\mid t=0} \in T_{x}(N) .
$$


The Killing vector field $X^{*}$ is right-invariant.

Lemma 2. The mapping defined by

$$
\mathfrak{n} \rightarrow \mathfrak{n}, \quad X \mapsto X+\frac{1}{2}[X, x]
$$

is a linear isomorphism.

Proof: Since the mapping is clearly linear, it is sufficient to prove that it is injective. In order to do this, we study the kernel of the mapping. Suppose that $X \in \mathfrak{n}$ satisfy the condition $X+\frac{1}{2}[X, x]=0$. Decompose $X$ as $X=X_{1}+X_{2}$ where $X_{1} \in \mathfrak{z}^{\perp}$ and $X_{2} \in \mathfrak{z}$, then $X_{1}+\left(X_{2}+\frac{1}{2}\left[X_{1}, x\right]\right)=0$. This implies $X_{1}=0$ and $X_{2}+\frac{1}{2}\left[X_{1}, x\right]=0$. Hence we have $X_{2}=0$, hence, $X=0$.

By the lemma above, we have $T_{x}(N)=\operatorname{span}\left\{X_{x}^{*} ; X \in \mathfrak{n}\right\}$ for any $x$ in $N$. The Killing vector field $X^{*}$ is an infinitesimal automorphism of $\phi$.

Lemma 3. Let $X$ be in $T_{0}(N)=\mathfrak{n}$. For a fixed $x \in N$, we have $X_{x}^{*}=\tilde{W}_{x}$, where we set $W=X+[X, x]$.

Proof: Since

$$
\begin{aligned}
\tilde{W}_{x} & =\frac{\mathrm{d}}{\mathrm{d} t}\left(x+t X+t[X, x]+\frac{t}{2}[x, X+[X, x]]\right)_{\mid t=0} \\
& =\frac{\mathrm{d}}{\mathrm{d} t}\left(x+t X+\frac{t}{2}[X, x]\right)_{\mid t=0}=X_{x}^{*}
\end{aligned}
$$

we have the assertion.

Lemma 4. Define a one-form $\eta_{a_{0}}$ on $N$ by

$$
\eta_{a_{0}}\left(X_{x}^{*}\right)=\left\langle[x, X], a_{0}\right\rangle, \quad X \in \mathfrak{n} .
$$

Then $\iota\left(X^{*}\right) \Omega_{a_{0}}=\mathrm{d}\left(\eta_{a_{0}}\left(X^{*}\right)\right)$ for any $X$ in $\mathfrak{n}$.

Proof: Let $X$ and $Y$ be in $\mathfrak{n}$. By Lemma 3, we have

$$
\begin{aligned}
\left(\iota\left(X_{x}^{*}\right) \Omega_{a_{0}}\right)\left(\tilde{Y}_{x}\right) & =\Omega_{a_{0}}\left(X_{x}^{*}, \tilde{Y}_{x}\right) \\
& =\Omega_{a_{0}}\left(\tilde{W}_{x}, \tilde{Y}_{x}\right) \\
& \left.=\Omega_{a_{0}}(X+[X, x]), Y\right) \\
& =\left\langle a_{0},[Y, X+[X, x]]\right\rangle=\left\langle a_{0},[Y, X]\right\rangle .
\end{aligned}
$$


Using the above equation, we have also

$$
\begin{aligned}
\mathrm{d}\left(\eta_{a_{0}}\left(X^{*}\right)\right)\left(\tilde{Y}_{x}\right) & =\tilde{Y}_{x}\left(\eta_{a_{0}}\left(X^{*}\right)\right) \\
& =\frac{\mathrm{d}}{\mathrm{d} t} \eta_{a_{0}}\left(X_{x+t Y+\frac{t}{2}[x, Y]}^{*}\right)_{\mid t=0} \\
& =\frac{\mathrm{d}}{\mathrm{d} t}\left\langle\left[x+t Y+\frac{t}{2}[x, Y], X\right], a_{0}\right\rangle \\
& =\left\langle[Y, X], a_{0}\right\rangle=\left(\iota\left(X_{x}^{*}\right) \Omega_{a_{0}}\right)\left(\tilde{Y}_{x}\right)
\end{aligned}
$$

Hence we get $\mathrm{d}\left(\eta_{a_{0}}\left(X^{*}\right)\right)=\iota\left(X^{*}\right) \Omega_{a_{0}}$.

Denote by $T_{x}(N) \rightarrow T_{0}(N) ; v \mapsto v_{\mathfrak{n}}$ the usual parallel translation in the Euclidean space $\mathfrak{n}$ : Take a curve $c(t)$ in $N$ such that $c(0)=x, \dot{c}(0)=v$. Then $v_{\mathfrak{n}}=$ $\frac{\mathrm{d}}{\mathrm{d} t}(c(t)-x)_{\mid t=0}$. The following lemma gives a relation between the two linear isomorphisms $L_{x}^{-1}: T_{x}(N) \rightarrow T_{0}(N)$ and $T_{x}(N) \rightarrow T_{0}(N), v \mapsto v_{\mathfrak{n}}$.

Lemma 5. $L_{x}^{-1} v=v_{\mathfrak{n}}-\frac{1}{2}\left[x, v_{\mathfrak{n}}\right] \quad$ for $\quad v \in T_{x}(N)$.

Proof: Take a curve $c(t)$ in $N$ such that $c(0)=x, \dot{c}(0)=v$. Then

$$
\begin{aligned}
L_{x}^{-1} v & =L_{-x} v=\frac{\mathrm{d}}{\mathrm{d} t}\left(-x+c(t)-\frac{1}{2}[x, c(t)]\right)_{\mid t=0} \\
& =\frac{\mathrm{d}}{\mathrm{d} t}\left(c(t)-x-\frac{1}{2}[x, c(t)-x]\right)_{\mid t=0}=v_{\mathfrak{n}}-\frac{1}{2}\left[x, v_{\mathfrak{n}}\right]
\end{aligned}
$$

Hence we have the assertion.

Similarly we define $T_{y}\left(\mathfrak{z}^{\perp}\right) \rightarrow T_{0}\left(\mathfrak{z}^{\perp}\right), u \mapsto u_{\mathfrak{z}^{\perp}}$ and $T_{z}(\mathfrak{z}) \rightarrow T_{0}(\mathfrak{z}), w \mapsto w_{\mathfrak{z}}$. Since $\mathfrak{z}$ is abelian, we have $L_{z}^{-1} w=w_{\mathfrak{z}}$ for $w \in T_{z}(\mathfrak{z})$. Hence we can write $w=w_{\mathfrak{z}}$. Let $x \in \mathfrak{n}$ and $v \in T_{x}(\mathfrak{n})$. Expressing $x$ and $v$ as $x=y+z$ and $v=v_{1}+v_{2}$, where $y \in \mathfrak{z}^{\perp}, z \in \mathfrak{z}, v_{1} \in T_{y}\left(\mathfrak{z}^{\perp}\right)$ and $v_{2} \in T_{z}(\mathfrak{z})$ we obtain

$$
L_{x}^{-1} v=\left(v_{1}\right)_{\mathfrak{z}^{\perp}}+\left(v_{2}-\frac{1}{2}\left[y,\left(v_{1}\right)_{\mathfrak{z}^{\perp}}\right]\right) .
$$

Proposition 6. Let $x(t)=y(t)+z(t)$ be a curve in $\mathfrak{n}$, where $y(t) \in \mathfrak{z}^{\perp}$ and $z(t) \in \mathfrak{z}$. Assume that $y(0)=0$. Then $x(t)$ describes the motion of a charged particle (3) if and only if

$$
\dot{y}(t)_{\mathfrak{z}^{\perp}}-\phi_{\dot{z}(0)+a_{0}} y(t)=\dot{y}(0), \quad \dot{z}(t)-\frac{1}{2}\left[y(t), \dot{y}(t)_{\mathfrak{z}^{\perp}}\right]=\dot{z}(0) .
$$


Proof: Taking the inner product of (3) and the Killing vector field $X^{*}$ for $X \in \mathfrak{n}$, we have

$$
\frac{\mathrm{d}}{\mathrm{d} t}\left\langle\dot{x}, X^{*}\right\rangle=\Omega\left(X^{*}, \dot{x}\right)=\left(\iota\left(X^{*}\right) \Omega\right)(\dot{x}) .
$$

Using Lemma 4 we find

$$
\frac{\mathrm{d}}{\mathrm{d} t}\left\langle\dot{x}, X^{*}\right\rangle=\left(\mathrm{d}\left(\eta\left(X^{*}\right)\right)\right)(\dot{x})=\frac{\mathrm{d}}{\mathrm{d} t} \eta\left(X_{x(t)}^{*}\right)
$$

Since $T_{x}(N)=\operatorname{span}\left\{X_{x}^{*} ; X \in \mathfrak{n}\right\}$, the equation (3) is equivalent to

$$
\frac{\mathrm{d}}{\mathrm{d} t}\left(\left\langle\dot{x}(t), X_{x(t)}^{*}\right\rangle-\eta\left(X_{x(t)}^{*}\right)\right)=0 .
$$

By the definition of $\eta$, we have

$$
\eta\left(X_{x(t)}^{*}\right)=\left\langle[x(t), X], a_{0}\right\rangle=\left\langle\phi_{a_{0}}(y(t)), X\right\rangle .
$$

Since $\langle$,$\rangle is left invariant$

$$
\begin{aligned}
\left\langle\dot{x}, X_{x(t)}^{*}\right\rangle & =\left\langle L_{x}^{-1} \dot{x}, L_{x}^{-1} X_{x}^{*}\right\rangle \\
& =\left\langle\dot{y}_{\mathfrak{z}^{\perp}}+\left(\dot{z}-\frac{1}{2}\left[y, \dot{y}_{\mathfrak{z}^{\perp}}\right]\right), X+[X, x]\right\rangle \\
& =\left\langle\dot{y}_{\mathfrak{z}^{\perp}}, X\right\rangle+\left\langle\dot{z}-\frac{1}{2}\left[y, \dot{y}_{\mathfrak{z}^{\perp}}\right], X+[X, x]\right\rangle
\end{aligned}
$$

where we have used Lemma 3 and equation (4). Hence the equation (3) is equivalent to

$$
\frac{\mathrm{d}}{\mathrm{d} t}\left(\left\langle\dot{y}_{\mathfrak{z}^{\perp}}-\phi_{a_{0}}(y), X\right\rangle+\left\langle\dot{z}-\frac{1}{2}\left[y, \dot{y}_{\mathfrak{z}^{\perp}}\right], X+[X, y]\right\rangle\right)=0 .
$$

Taking $X \in \mathfrak{z}$, we have

$$
\dot{z}(t)-\frac{1}{2}\left[y(t), \dot{y}(t)_{\mathfrak{z}^{\perp}}\right]=\dot{z}(0)
$$

where we have used the initial condition $y(0)=0$. Next, taking $X \in \mathfrak{z}^{\perp}$, we have

$$
\frac{\mathrm{d}}{\mathrm{d} t}\left(\left\langle\dot{y}_{\mathfrak{z}^{\perp}}-\phi_{a_{0}}(y), X\right\rangle+\langle\dot{z}(0),[X, y]\rangle\right)=0 .
$$

Taking into account the initial condition $y(0)=0$, we finally have

$$
\dot{y}(t)_{\mathfrak{z}^{\perp}}-\phi_{\dot{z}(0)+a_{0}} y(t)=\dot{y}(0) .
$$


Proposition 7. The motion of a charged particle (3) with $y(0)=0$ is given by the equations

$$
\begin{aligned}
& y(t)=\exp t \phi_{\dot{z}(0)+a_{0}} \int_{0}^{t} \exp \left(-t \phi_{\dot{z}(0)+a_{0}}\right) \dot{y}(0) \mathrm{d} t \\
& z(t)=z(0)+t \dot{z}(0)+\frac{1}{2} \int_{0}^{t}\left[y(t),\left(\exp t \phi_{\dot{z}(0)+a_{0}}\right) \dot{y}(0)\right] \mathrm{d} t
\end{aligned}
$$

Proof: Using the first equation of (5) with $y(0)=0$, we have

$$
y(t)=\exp t \phi_{\dot{z}(0)+a_{0}} \int_{0}^{t} \exp \left(-t \phi_{\dot{z}(0)+a_{0}}\right) \dot{y}(0) \mathrm{d} t .
$$

Hence

$$
\phi_{\dot{z}(0)+a_{0}} y(t)=\left(\exp t \phi_{\dot{z}(0)+a_{0}}-1\right) \dot{y}(0)
$$

which implies that

$$
\phi_{\dot{z}(0)+a_{0}} y(t)+\dot{y}(0)=\left(\exp t \phi_{\dot{z}(0)+a_{0}}\right) \dot{y}(0) .
$$

Using the second and the first equation from (5)

$$
\begin{aligned}
z(t) & =z(0)+t \dot{z}(0)+\frac{1}{2} \int_{0}^{t}\left[y(t), \dot{y}(t)_{\mathfrak{z}^{\perp}}\right] \mathrm{d} t \\
& =z(0)+t \dot{z}(0)+\frac{1}{2} \int_{0}^{t}\left[y(t), \phi_{\dot{z}(0)+a_{0}} y(t)+\dot{y}(0)\right] \mathrm{d} t \\
& =z(0)+t \dot{z}(0)+\frac{1}{2} \int_{0}^{t}\left[y(t),\left(\exp t \phi_{\dot{z}(0)+a_{0}}\right) \dot{y}(0)\right] \mathrm{d} t
\end{aligned}
$$

Hence we get the assertion.

When $\phi_{\dot{z}(0)+a_{0}}=0$, then, using the above Proposition, we get $y(t)=t \dot{y}(0)$ and

$$
z(t)=z(0)+t \dot{z}(0)+\frac{1}{2} \int_{0}^{t}[t \dot{y}(0), \dot{y}(0)] \mathrm{d} t=z(0)+t \dot{z}(0) .
$$

Lemma 8. The equation of motion (3) implies the following relation

$$
\frac{\mathrm{d}}{\mathrm{d} t}\left(\left\langle z(t), \dot{z}(0)+a_{0}\right\rangle+\frac{1}{2}\langle y(t), \dot{y}(0)\rangle\right)=|\dot{z}(0)|^{2}+\left\langle\dot{z}(0), a_{0}\right\rangle+\frac{1}{2}\left|\dot{y}_{\mathfrak{z}^{\perp}}\right|^{2} .
$$

Proof: Taking the inner product of the second equation of (5) with $\dot{z}(0)+a_{0}$, we have

$$
\left\langle\dot{z}, \dot{z}(0)+a_{0}\right\rangle-\frac{1}{2}\left\langle\left[y, \dot{y}_{\mathfrak{z}^{\perp}}\right], \dot{z}(0)+a_{0}\right\rangle=|\dot{z}(0)|^{2}+\left\langle\dot{z}(0), a_{0}\right\rangle .
$$


Using equation (5) again produces

$$
\begin{aligned}
\left\langle\left[y, \dot{y}_{\mathfrak{z}^{\perp}}\right], \dot{z}(0)+a_{0}\right\rangle & =\left\langle\phi_{\dot{z}(0)+a_{0}} y, \dot{y}_{\mathfrak{z}^{\perp}}\right\rangle \\
& =\left\langle\dot{y}_{\mathfrak{z}^{\perp}}-\dot{y}(0), \dot{y}_{\mathfrak{z}^{\perp}}\right\rangle \\
& =\left|\dot{y}_{\mathfrak{z}^{\perp}}\right|^{2}-\left\langle\dot{y}_{\mathfrak{z}^{\perp}}, \dot{y}(0)\right\rangle=\left|\dot{y}_{\mathfrak{z}^{\perp}}\right|^{2}-\frac{\mathrm{d}}{\mathrm{d} t}\langle y(t), \dot{y}(0)\rangle .
\end{aligned}
$$

Hence

$$
\frac{\mathrm{d}}{\mathrm{d} t}\left(\left\langle z(t), \dot{z}(0)+a_{0}\right\rangle+\frac{1}{2}\langle y(t), \dot{y}(0)\rangle\right)=|\dot{z}(0)|^{2}+\left\langle\dot{z}(0), a_{0}\right\rangle+\frac{1}{2}\left|\dot{y}_{\mathfrak{z}^{\perp}}\right|^{2} .
$$

Applying the lemma above for geodesics, we can re-demonstrate the following theorem of Naitoh-Sakane.

Theorem 9. (Naitoh-Sakane [4, Corrolary 3.3]) Every geodesic in any simply connected two-step nilpotent Lie group with a left-invariant Riemannian metric does not intersect itself.

Proof: Let $x(t)=y(t)+z(t) \in N$ be a geodesic with $y(0)=0$. Applying Lemma 8 with $a_{0}=0$

$$
\frac{\mathrm{d}}{\mathrm{d} t}\left(\langle z(t), \dot{z}(0)\rangle+\frac{1}{2}\langle y(t), \dot{y}(0)\rangle\right)=|\dot{z}(0)|^{2}+\frac{1}{2}\left|\dot{y}_{\mathfrak{z}^{\perp}}\right|^{2}>0 .
$$

Hence $\langle z(t), \dot{z}(0)\rangle+\frac{1}{2}\langle y(t), \dot{y}(0)\rangle$ is monotone increasing. Thus $x(t)$ is not periodic. Since we have already proved that $x(t)$ is simple by Theorem 1 , we get the assertion.

The author thinks that the above proof is easier than the original proof of NaitohSakane.

\section{Charged Particles in $H$-type Lie Groups}

In this section, we study the motion of a charged particle in a simply connected $H$-type Lie group. First we review the definition of $H$-type Lie algebra according to Kaplan. Let $(U,\langle\rangle$,$) and (V,\langle\rangle$,$) be finite-dimensional real vector spaces with$ inner products $\langle$,$\rangle . Denote by \operatorname{End}(V)$ the vector space consisting of all linear transformations on $V$. We assume that there exists a linear mapping $j: U \rightarrow$ $\operatorname{End}(V)$ such that

$$
j(a)^{2}=-|a|^{2} I, \quad|j(a) x|=|a||x|, \quad a \in U, \quad x \in V .
$$


In other words, $V$ is a Clifford module over the Clifford algebra generated by $U$. By (6) we have

$$
\begin{aligned}
& \langle j(a) x, j(b) x\rangle=\langle a, b\rangle|x|^{2}, \quad\langle j(a) x, j(a) y\rangle=|a|^{2}\langle x, y\rangle \\
& \langle j(a) x, y\rangle+\langle x, j(a) y\rangle=0, \quad a, b \in U, \quad x, y \in V .
\end{aligned}
$$

Define a bi-linear mapping [,] $V \times V \rightarrow U$ via the formula

$$
\langle a,[x, y]\rangle=\langle j(a) x, y\rangle, \quad a \in U, \quad x, y \in V .
$$

Then $[$,$] is alternative. Substituting j(b) x$ into $y$, we have

$$
\langle a,[x, j(b) x]\rangle=\langle j(a) x, j(b) x\rangle=\langle a, b\rangle|x|^{2} .
$$

Hence

$$
[x, j(b) x]=|x|^{2} b, \quad b \in U, \quad x \in V .
$$

We denote by $\mathfrak{n}=U \oplus V$ the orthogonal direct sum of $U$ and $V$, and define a Lie algebra structure on $\mathfrak{n}$ by

$$
[a+x, b+y]=[x, y] \in U, \quad a, b \in U, \quad x, y \in V .
$$

Then the Lie algebra $\mathfrak{n}$ is called $H$-type. Since the $H$-type Lie algebra $\mathfrak{n}$ is a kind of two-step nilpotent Lie algebra with an inner product, we can define a Lie group structure on $\mathfrak{n}$ with a left-invariant Riemannian metric, whose Lie algebra is $\mathfrak{n}$ itself as we mentioned in the previous section. For $a_{0} \in U$, we consider the equation

$$
\nabla_{\dot{x}} \dot{x}=j\left(a_{0}\right) \dot{x}
$$

of motion of a charged particle. If we express its trajectory as $x(t)=y(t)+z(t)$ where $y(t) \in V, z(t) \in U$, then (9) is equivalent to

$$
\dot{y}(t)_{V}-j\left(\dot{z}(0)+a_{0}\right) y(t)=\dot{y}(0)
$$

where $T_{y}(V) \rightarrow V, w \mapsto w_{V}$ denotes the usual parallel translation in $V$. Here we have used equation (5).

Theorem 10. Let $x(t)=y(t)+z(t) \in N$ (where $y(t) \in V, z(t) \in U)$ is a motion of a charged particle (9) with $x(0)=0$.

1) When $\dot{z}(0)+a_{0}=0$, then $x(t)=t \dot{x}(0)$.

2) When $\dot{z}(0)+a_{0} \neq 0$, then

$$
\begin{aligned}
& y(t)=\frac{\sin \left(t\left|\dot{z}(0)+a_{0}\right|\right)}{\left|\dot{z}(0)+a_{0}\right|} \dot{y}(0)+\frac{1-\cos \left(t\left|\dot{z}(0)+a_{0}\right|\right)}{\left|\dot{z}(0)+a_{0}\right|^{2}} j\left(\dot{z}(0)+a_{0}\right) \dot{y}(0) \\
& z(t)=t \dot{z}(0)+\frac{t|\dot{y}(0)|^{2}}{2\left|\dot{z}(0)+a_{0}\right|^{2}}\left(\dot{z}(0)+a_{0}\right)-\frac{\sin \left(t\left|\dot{z}(0)+a_{0}\right|\right)}{2\left|\dot{z}(0)+a_{0}\right|^{3}}|\dot{y}(0)|^{2}\left(\dot{z}(0)+a_{0}\right) .
\end{aligned}
$$


The curve $y(t)$ is a circle in $V$. The motion of a charged particle is periodic if and only if

$$
a_{0}=-\left(\frac{|\dot{y}(0)|^{2}}{2|\dot{z}(0)|^{2}}+1\right) \dot{z}(0) .
$$

In this case $x(t)$ is an elliptic motion.

Remark 11. When $x(t)$ is a geodesic, the condition $a_{0}=0$ implies the theorem of Kaplan [3].

Proof: 1) is clear from (10). We will show 2). Using the first equation of (10), we have

$$
y(t)=\frac{\sin \left(t\left|\dot{z}(0)+a_{0}\right|\right)}{\left|\dot{z}(0)+a_{0}\right|} \dot{y}(0)+\frac{1-\cos \left(t\left|\dot{z}(0)+a_{0}\right|\right)}{\left|\dot{z}(0)+a_{0}\right|^{2}} j\left(\dot{z}(0)+a_{0}\right) \dot{y}(0)
$$

which implies that

$$
\dot{y}(t)_{V}=\cos \left(t\left|\dot{z}(0)+a_{0}\right|\right) \dot{y}(0)+\frac{\sin \left(t\left|\dot{z}(0)+a_{0}\right|\right)}{\left|\dot{z}(0)+a_{0}\right|} j\left(\dot{z}(0)+a_{0}\right) \dot{y}(0) .
$$

Using the equation above, we have

$$
\left[y(t)_{V}, \dot{y}(t)\right]=\frac{1-\cos \left(t\left|\dot{z}(0)+a_{0}\right|\right)}{\left|\dot{z}(0)+a_{0}\right|^{2}}\left[\dot{y}(0), j\left(\dot{z}(0)+a_{0}\right) \dot{y}(0)\right] .
$$

Further the second equation of (10) gives

$$
\begin{aligned}
\dot{z}(t) & =\dot{z}(0)+\frac{1-\cos \left(t\left|\dot{z}(0)+a_{0}\right|\right)}{2\left|\dot{z}(0)+a_{0}\right|^{2}}\left[\dot{y}(0), j\left(\dot{z}(0)+a_{0}\right) \dot{y}(0)\right] \\
& =\dot{z}(0)+\frac{1-\cos \left(t\left|\dot{z}(0)+a_{0}\right|\right)}{2\left|\dot{z}(0)+a_{0}\right|^{2}}\left(\dot{z}(0)+a_{0}\right)|\dot{y}(0)|^{2}
\end{aligned}
$$

where we have used the equation (8). Since

$$
\begin{aligned}
y(t)-\frac{1}{\left|\dot{z}(0)+a_{0}\right|} j\left(\frac{\dot{z}(0)+a_{0}}{\left|\dot{z}(0)+a_{0}\right|}\right) \dot{y}(0)=\frac{\sin \left(\left|\dot{z}(0)+a_{0}\right| t\right)}{\left|\dot{z}(0)+a_{0}\right|} \dot{y}(0) \\
-\frac{\cos \left(\left|\dot{z}(0)+a_{0}\right| t\right)}{\left|\dot{z}(0)+a_{0}\right|} j\left(\frac{\dot{z}(0)+a_{0}}{\left|\dot{z}(0)+a_{0}\right|}\right) \dot{y}(0)
\end{aligned}
$$

the curve $y(t)$ is a circle in $V$ whose center is $\frac{1}{\left|\dot{z}(0)+a_{0}\right|} j\left(\frac{\dot{z}(0)+a_{0}}{\left|\dot{z}(0)+a_{0}\right|}\right) \dot{y}(0)$ and the radius is $\frac{|\dot{y}(0)|}{\left|\dot{z}(0)+a_{0}\right|}$. The periodic condition is as follows

$$
\begin{aligned}
x(t) \text { is periodic } & \Leftrightarrow \dot{z}(0)+\frac{|\dot{y}(0)|^{2}}{2\left|\dot{z}(0)+a_{0}\right|^{2}}\left(\dot{z}(0)+a_{0}\right)=0 \\
& \Leftrightarrow a_{0}=-\left(\frac{|\dot{y}(0)|^{2}}{2|\dot{z}(0)|^{2}}+1\right) \dot{z}(0) .
\end{aligned}
$$


In this case, since

$$
\begin{array}{r}
x(t)+\frac{2|\dot{z}(0)|}{|\dot{y}(0)|^{2}} j\left(\frac{\dot{z}(0)}{|\dot{z}(0)|}\right) \dot{y}(0)=\frac{2|\dot{z}(0)|}{|\dot{y}(0)|^{2}}\left(\sin \left(\frac{|\dot{y}(0)|^{2}}{2|\dot{z}(0)|} t\right)(\dot{y}(0)+\dot{z}(0))\right. \\
\left.+\cos \left(\frac{|\dot{y}(0)|^{2}}{2|\dot{z}(0)|} t\right) j\left(\frac{\dot{z}(0)}{|\dot{z}(0)|}\right) \dot{y}(0)\right)
\end{array}
$$

the curve $x(t)$ is an elliptic such that the ratio of the long axis to the short axis is equal to $\sqrt{|\dot{y}(0)|^{2}+|\dot{z}(0)|^{2}} /|\dot{y}(0)|$.

\section{Acknowledgments}

The author was partially supported by the Japan Society for Promotion of Science through a Grant-in-Aid for Scientific Research (\# 22540108).

\section{References}

[1] Helgason S., Differential Geometry, Lie Groups and Symmetric Spaces, Academic Press, New York 1978.

[2] Ikawa O., Motion of Charged Particles From the Geometric View Point, J. Geom. Symm. Phys. 18 (2010) 23-47.

[3] Kaplan A., Riemannian Nilmanifiolds Attached to Clifford Modules, Geometriae Dedicata 11 (1981) 127-136.

[4] Naitoh H. and Sakane Y., On Conjugate Points of a Nilpotent Lie Group, Tsukuba J. Math. 5 (1981) 143-152.

Osamu Ikawa

Department of General Education

Fukushima National College of Technology

Iwaki, Fukushima, 970-8034

JAPAN

E-mail address: ikawa@fukushima-nct.ac.jp 